\title{
Slow and steady wins the race? No signs of reduced welfare in smallerbroiler breeder hens at four weeks of age
}

Caroline Lindholm, A Calais, J Jönsson, N. Yngwe, E. Berndtson, E. Hult and Jordi Altimiras

\section{Linköping University Post Print}

\section{Tweet}

N.B.: When citing this work, cite the original article.

Original Publication:

Caroline Lindholm, A Calais, J Jönsson, N. Yngwe, E. Berndtson, E. Hult and Jordi Altimiras, Slow and steady wins the race? No signs of reduced welfare in smallerbroiler breeder hens at four weeks of age, 2015, Animal Welfare, (24), 447-454.

http://dx.doi.org/10.7120/09627286.24.4.447

Copyright: Universities Federation for Animal Welfare (UFAW)

http://www.ufaw.org.uk/

Postprint available at: Linköping University Electronic Press

http://urn.kb.se/resolve?urn=urn:nbn:se:liu:diva-122024 
Slow and steady wins the race? No signs of reduced welfare

in smaller broiler breeder hens

at four weeks of age

[Growth and welfare in four-week broiler breeders]

C Lindholm ${ }^{1}$, A Calais ${ }^{1}$, J Jönsson ${ }^{1}$, N Yngwe ${ }^{2}$, E Berndtson ${ }^{2}$, E Hult ${ }^{2} \&$ J Altimiras ${ }^{1 *}$

1. AVIAN Behavioural Genomics and Physiology group, IFM, Linköping University, Linköping, Sweden

2. SweHatch AB, Flyinge, Sweden

* Corresponding author: jordi.altimiras@ liu.se; phone: +46 132858 24;

postal address: Jordi Altimiras, Linköping university, IFM, 58183 Linköping, Sweden 


\section{Abstract (max 250 words)}

Broiler breeder chickens are commonly reared under strict feed-restriction regimes to reduce obesity-induced health and fertility problems during adult life, and are assumed to experience a reduced welfare due to the resulting hunger. In these conditions feed competition could influence the growth rate, so that the individuals falling behind in growth would experience more stress and hunger. We hypothesized that these chickens are poor competitors due to a reactive coping style and experience a further reduced welfare situation before size-sorting ("grading") at 4 weeks of age. Our results from open field, tonic immobility and home pen activity monitoring show signs of lower fear and higher home-pen activity levels in smaller hens and do not support the idea of reactive coping. $\mathrm{H} / \mathrm{L}$ ratios of smaller hens were also found to be lower, indicating less stress in these birds. Dissections of smaller and larger four-week breeder hens may offer an explanation in the form of a relatively larger gastrointestinal tract in smaller birds. We argue that this is a form of habituation to restricted feeding, offering these birds a physiological stress coping mechanism, and that low early growth rate may not always be a sign of poorer welfare in broiler breeders.

\section{Keywords (six, listed alphabetically)}

Animal welfare, broiler breeders, chicken, feed restriction, growth, stress.

\section{Introduction}

During the last 50 years broiler chickens have been selected for increasingly efficient meat production, leading to an astonishing $400 \%$ increase in growth rate while decreasing feed conversion ratios (FCR) by 50\% (Zuidhof et al 2014). These improvements in production traits do however come at a price for the parental chicken generation, the broiler breeders. The high growth rate of broilers is not only coupled to high rates of cardiovascular and skeletal 
pathologies, but also to reduced fertility due to follicular hypersensitivity to local growth factors (GFs), premature HPA axis maturation and obesity-induced lipotoxicity in ovarian tissues (Bruggeman et al 1999; Decuypere et al 2002; Chen et al 2006). To overcome these problems, broiler breeders are commonly reared under feed restriction regimes which may reach feeding levels as low as a third of ad libitum intake during the most intense periods (de Jong et al 2002). The hunger and feeding frustration experienced by broiler breeders are often acknowledged as one of the major animal welfare problems of our time but there is also evidence that the stress can be alleviated to some extent by good rearing practices, such as litter-based flooring which allows for natural foraging behaviours and gradual habituation to the restriction regime (Hocking et al $1993 ; 1996)$.

Feed restriction in itself has been found to decrease flock uniformity (Zuidhof et al 1995), and the rushed eating and increased competition for feed that go along with it could justify the increased heterogeneity (Bennett \& Leeson 1989; Zuidhof et al 1995). This heterogeneity in growth is expected to arise due to unequal distribution of feed between individuals, which specifically leads some animals to fall behind in growth (Aviagen 2013). According to the "uniformity is healthy" hypothesis (cf Dawkins et al 2013), individual animals experiencing adverse conditions will increase physiological and behavioural heterogeneity within their flock and so a lack of uniformity can be interpreted as a sign of welfare problems (Zuidhof et al 1995; Dawkins et al 2013). Flock uniformity in growth is also one of the major quality measures used in commercial broiler breeder rearing, where the aim is to keep the coefficient of variance $(\mathrm{CV})$ for body mass low to facilitate animal management (Aviagen 2013). Based on this, we would expect lower welfare in the under-feeders, ie the smaller animals, which may be experiencing an intensified feed-restriction. Commercial rearing farms attempt to alleviate this problem by size-sorting 
("grading") the animals at 4 weeks of age, putting the smaller birds in a separate pen with less feed competition.

It is well known that individual animals have different abilities and strategies for coping with stressful situations. The coping style of an individual is influenced by both genotype and experiences during early development, but is then rather fixed (Koolhaas et al 1999). While wild animals tend to group into two distinct coping styles, domestic animals typically show a normal distribution of coping styles with only the extreme animals clearly demonstrating the classical proactive and reactive coping styles (Koolhaas et al 1999). Proactive animals are typically identified as more active, aggressive and routine-forming while reactive animals are more passive and flexible to new situations (Koolhaas et al 1999). In birds, proactive individuals have been shown to exhibit lower levels of corticosterone (CORT) in response to stressors, higher locomotor activity and a shorter duration of tonic immobility (Korte et al 1997). We chose to use the open field (OF) and tonic immobility (TI) tests to examine behavioural differences between large and small birds as these are well-known and well validated for quantifying fearfulness in poultry (Forkman et al 2007). They are also reasonably easy to set up in a farm setting, and worked well in pilot tests with laboratory-raised chickens. As we expected the commercially reared chickens to have received very little socialisation and were concerned that the other tests may prove too stressful, we also studied their daily activity levels when left undisturbed and in connection with a stressful event in the home pen.

Thus we hypothesized that (1) broiler breeder chickens within the same commercial rearing flock will experience quantifiably different welfare situations and that (2) animals exhibiting a reactive coping style would be less efficient at competing for a limited amount of feed, experience an increased feed-restriction and thereby a reduced individual welfare situation which would (3) 
lead to these animals being considerably smaller than average before undergoing size-sorting at 4 weeks of age. To test these hypotheses, we investigated the $\mathrm{H} / \mathrm{L}$ ratios, internal organ sizes and behaviours of four-week-old commercially reared broiler breeder hens of a small or large body size prior to size-sorting.

\section{Materials and methods}

Experiments were carried out using Ross 308 broiler breeder hens before the first size-sorting around 4 weeks of age. The chickens were reared on a commercial farm owned by SweHatch AB according to industry standards and Swedish legislation. The chickens were kept in flocks of approximately 3500 hens per pen from hatch until the time of the experiment. Two batches of chickens were used: one for the behaviour tests and one for the blood sampling and dissections. A batch of chickens is here defined as animals originating from the same grand-parental flock that have also been hatched and reared together. At the time of the experiments, all chicks were on a 8L:16D lighting schedule with feed distributed daily just after artificial dawn at $0700 \mathrm{~h}$. Feed was distributed evenly across the floor using an automated feed spinner which the animals had been gradually habituated to while 3-12 days of age. Feed restriction was gradually introduced from the age of 1 week. Ethical permission for the experiments was granted to J Altimiras by the regional ethical committee of Malmö/Lund (diary number M 71-14).

\section{Behaviour testing}

Behavioural tests were performed on 124 broiler breeder hens over the course of 3 days at an age of 26-28 days old. Animals were chosen randomly in the pen and assigned to two experimental groups if their body mass were > 1 standard deviation heavier than the mean (group L as "large") or > 1 standard deviation lighter than the mean (group S as "small"). Mean body mass was 
updated daily and based on the automatic in-farm monitoring system (Big Dutchman, Calveslage, Germany) and standard deviation was based on the weight distribution recorded by the farmer during the previous week (coefficient of variance: $15.1 \%$ ). Mean weights were $604 \mathrm{~g}$ (SD: 25) and $383 \mathrm{~g}$ (SD: 21) for the two groups respectively. Birds were collected from their home pen in groups of four (two large and two small) just before the experiments and briefly kept in separate compartments in the same box. Each group of four animals were simultaneously subjected to an open field (OF) test (arena $1.2 \times 0.8 \mathrm{~m}$ ) during $5 \mathrm{~min}$ and then returned to the box. Tonic immobility (TI) was induced by $15 \mathrm{~s}$ of back restraint, and each bird given up to three induction trials. TI was interrupted after $5 \mathrm{~min}$. The tested birds were then colour-marked to avoid resampling of the same birds, and returned to their home pen. Vocalisations were recorded on a digital voice recorder (Olympus, Tokyo, Japan) during the OF test and analysed manually for number, durations and types of vocalisations (Collias \& Joos 1953; Marx et al 2001).

Hens from the same batch but reared in another pen were monitored for daily activity levels using a tri-axial accelerometer device (MotionWatch 8, CamNTech, Cambridge, UK) worn on the back and attached by elastic bands around the base of each wing. No hens from this pen were used in the $\mathrm{OF}$ and $\mathrm{TI}$ tests, to avoid unintentional experiment-induced stress events. The devices were attached to three small and three large birds every afternoon just before artificial dusk on three consecutive days for a total of 18 birds monitored. The devices were in place for approximately $23 \mathrm{~h}$. Every day at noon (1200h) the monitored birds were collected and stressed by being placed in a freely hanging mesh bag for 3 min before returning to the flock. All birds were previously naïve to this stressor, and tested birds were marked to avoid re-sampling. Due to a hardware malfunction, data from one of the small birds had to be discarded.

\section{Blood smears and dissections}


Blood samples $(1 \mathrm{ml})$ were drawn from the ulnar vein of 59 broiler breeder hens at 33 days of age using $1 \mathrm{ml}$ syringes with EDTA as an anticoagulant. Blood sampling was done during the dark period $(1530 \mathrm{~h}-0130 \mathrm{~h})$ and under conditions of minimal blue lighting to disturb the animals as little as possible. Small and large animals were sampled alternately to avoid time-based variations between groups. Blood was used immediately to produce two blood smears per individual. Hens were chosen so that 28 individuals were $>1$ standard deviation heavier than the target weight ("large") and 31 individuals > 1 standard deviation lighter than the target weight ("small"). Live body masses of the two groups averaged $626 \mathrm{~g}$ (SD: 24) and $446 \mathrm{~g}$ (SD: 24) respectively. After drying, blood smears were fixed by 30 s methanol immersion, air-dried and stained with Giemsa solution (HistoLab Products AB, Göteborg, Sweden) for 30 min. Cells were counted manually under 100x magnification until a total of 100 white blood cells was reached, and the number of heterophils divided by the number of lymphocytes to produce $\mathrm{H} / \mathrm{L}$ ratios (cf Gross \& Siegel 1983). All blood smears were counted in duplicates with the observer blind to the treatment and the duplicate. Birds were euthanized by decapitation and dissected to obtain the weights of the ventricles (referred to as "heart" in the results), liver, spleen, both lungs, both kidneys, crop, proventriculus, gizzard and pancreas as well as the lengths of the duodenum, jejunum, ileum, large intestine (including rectum but not cloaca) and the average length of the caeca. The mass of residual feed left in the upper gastrointestinal tract and of the fat surrounding the gizzard (which we will call "abdominal fat" even if no effort was made to dissect out all fat surrounding abdominal organs) was subtracted from the weight of the whole animal to estimate the lean body mass.

\section{Statistics}


Organ sizes were normalised to lean body mass (LBM) and are reported as either \% LBM or length/LBM. Results were analysed using either the Log-Rank test when comparing KaplanMeier survival curves (Figures $2 \mathrm{~b}$ and 3 ) or the Student's t-test (two-tailed) when comparing two means. Results were considered significant when $P<0.05$. In all comparisons made, the birds being compared were from the same batch and reared in the same pen since hatch. Where present, errors bars denote standard error of the mean. All statistical analyses were performed using MiniTab® 17 software (MiniTab Inc, State College, PA).

\section{Results}

$\mathrm{H} / \mathrm{L}$ ratios were found to be significantly higher in larger birds, averaging 0.46 compared to 0.37 in the smaller group (Figure 1a). There was also a small, but significant positive correlation between $\mathrm{H} / \mathrm{L}$ ratio and body weight $\left(P=0.01, \mathrm{R}^{2}=9.3 \%\right.$; data not shown).

The farm-raised birds were very hesitant to move in the OF and differences in movement could not be seen between the groups (data not shown). Analysis of the vocalisations recorded during OF testing does however show that smaller birds vocalised significantly more during the test (average 115 vocalisations in $\mathrm{S}$ and 65 vocalisations in $\mathrm{L}$ ) and started vocalising on average $30 \mathrm{~s}$ earlier, although the latency to vocalise did not quite reach significance at $P=0.057$ (Figure 2). All recorded vocalisations were identified as either distress calls or fear trills (Collias \& Joos 1953; Marx et al 2001). Smaller birds also proved considerably more resistant to TI induction and only 33 small birds entered TI compared to 51 large birds (Figure 3a). Small birds that entered TI were also quicker to start moving their heads (Figure 3b), and spent significantly less total time being immobile (Figure $3 b$ ). When monitoring the level of daily activity in the home pen, smaller chicks were consistently, although not always significantly, more active than larger 
birds. In the afternoons smaller chicks were significantly more active than their larger conspecifics, although this difference is confounded by large individual variations just after being subjected to a stressful event (Figure 4).

While small and large birds had lungs and kidneys of comparable relative size (Figure 1b, see also Table 1), the hearts and livers of small animals were relatively larger. This pattern was also seen for the organs of the gastrointestinal tract, which all turned out to be relatively larger in small birds (Figure 5). The difference is most accentuated for the relative lengths of the different parts of the small intestine (duodena, jejuna and ilea), where large and small birds have very similar intestinal lengths in absolute terms (total average 166.3 (SD 14.3) and $161.3 \mathrm{~cm}$ (SD 13.8) respectively). There was no significant difference in the proportional sizes of the three parts, with the length of the small intestine being made up of approximately $17 \%$ duodenum, $41 \%$ jejunum and $42 \%$ ileum in both groups. Only the amount of abdominal fat was found to be relatively larger in large birds. While measuring actual feed intake was beyond the scope of this study, it is worth noting that there was no difference in the absolute amount of feed (24.2 g, SD 14.7) retained in the upper gastrointestinal tract in small and large birds at this time of day.

\section{Discussion}

We hypothesized that smaller hens were falling behind in growth due to a reactive coping style, which would be indicated by lower activity levels, less vocalisations and longer durations of TI. All of our behavioural data does however point in the opposite direction, with smaller hens being generally more active and less fearful than their larger counterparts. The responses to the mesh bag restraint in the home pen are also highly variable within both groups, and do not seem to support the idea of distinct coping style differences between large and small broiler breeder hens 
at 4 weeks of age. For this reason we chose not to continue the evaluation of coping styles in these birds.

Whereas we first took an interest in locomotor activity as an indicator of coping style, other reasons for higher activity levels have also been suggested. For example, increased activity level may be an indicator of feeding frustration (Mench 2002), which would not be unexpected in these birds as we expect feed competition to be a major driver of growth differences. The amount of feed found in the upper gastrointestinal tract upon dissection does not offer any support for the notion that smaller birds may be less successful feeders, as birds from both groups were found with comparable amounts of feed still in their crop, proventriculus and gizzard. However, this may not be representative for their actual feed intake as Katanbaf et al (1989) reported that feedstoring time was the result of habituation to a certain length of fasting time and did not follow the level of feed restriction.

Hocking et al (1996) also found a positive correlation between duration of TI and body weight, and argued that this may partly be an artefact as large birds may be less mobile than their smaller conspecifics. Although our birds are probably small enough that this is not an issue we have also reported latency to move the head, which is expected to be less affected by body size and in our case follows the total duration of TI very closely (Figure 3b). Fearfulness has previously often been reported to be negatively correlated with growth and productivity parameters (Jones et al 1997), although Skinner-Noble et al (2003) were unable to find this pattern in broilers and we appear to find the opposite. This may be a broiler-specific trait, perhaps as a result of the extreme selection for growth traits in these birds that may have dissociated traits that are otherwise correlated and possibly further affected by the feeding frustration experienced by broiler breeders. 
The raised $\mathrm{H} / \mathrm{L}$ ratios in large birds indicate higher circulating levels of corticosterone in these birds, and may be indicative of a higher experienced level of feed restriction in these birds (cf Gross \& Siegel 1983; Hocking et al 1993), in contradiction with our behavioural results. The increased CORT levels inducing an elevation in H/L ratios may however also be the result of higher metabolic demands in larger birds, as suggested by de Jong et al (2002). This explanation is further supported by the slight positive relationship found between H/L ratios and body mass in our data. Contradictingly, the enlarged hearts and livers seen in the smaller birds upon dissection would rather point in the opposite direction with a higher metabolic load possibly experienced by smaller birds.

While we cannot make any conclusions about the amount of feed ingested by different birds, our dissection data suggests that slower growth in some birds may not only be the result of eating less but could also be the result of growing differently. The increased sizes of gastrointestinal organs in smaller birds may indicate that these individuals are investing a larger part of their nutrient intake into building expensive gastrointestinal tissues rather than bulky muscle and fat tissue. This investment would then be expected to pay off in the long run, assuming that these larger GI tracts will actually result in higher GI uptake capabilities. It has previously been shown that the high growth rates in broilers are the result of longer and heavier intestines, rather than a qualitative change in tissue composition (Jackson \& Diamond 1996). Following this line of reasoning, enlarged GI tracts may be considered a form of predictive adaptive response during feed restriction, letting the animal make the most of the resources available to it. This is similar to the enlarged GI organs found by Pinchasov et al (1985) in chickens under high gastrointestinal strain (fasting). They also found a positive correlation between lipogenesis (partly indicated by liver hypertrophy) and gastrointestinal capacity in these birds, as well as relatively larger GI 
tracts in lighter birds. This matches our results very closely, and supports the idea that this is a physiological coping strategy under nutritionally challenging conditions. Katanbaf et al (1989) also reported enlarged intestines in broiler breeders undergoing feed-restriction compared to $\mathrm{ad}$ libitum-fed ones. While broilers raised on ad lib feeding may not represent normal physiology (de Jong et al 2002), Katanbaf et al (1989) also found larger pancreases and gizzards in the restricted birds and higher levels of carcass lipids in the ad libitum group, but no difference in heart or liver size. The hypothesis that nutritional challenges trigger an improved GI efficiency in some birds is further supported by the findings of Skinner-Noble et al (2003) that birds starting an FCR trial lighter tended to show better feed conversion ratios than initially heavier birds.

Improved feed conversion ratios have also been previously linked to low adiposity (Leenstra \& Pit 1988) and to increased egg production (Zuidhof et al 1995). Thus, if the slow-growing chicks are able to retain their enlarged GI tracts into the laying phase they may also turn out to be more productive breeders. Whether that is the case or not is however outside the scope of this study.

While we have generally interpreted the recorded vocalisations as signs of fear, distress calls are also used by young chicks to prompt social contact (Marx et al 2001). An alternative interpretation might thus be that smaller chicks are more socially motivated than larger chicks, which could potentially alleviate the social stress of being reared in a large flock where social tolerance is expected to be important and may in itself help reduce stress levels in these birds (Marin et al 2001; Kikusui et al 2006; Estevez et al 2007).

\section{Conclusion and animal welfare implications}

Feed-restricted broiler breeders of different body weights at 4 weeks of age are not different in terms of coping style, but smaller birds show traits that may be adaptive to feed-restriction, 
possibly enhancing both their welfare status and productivity traits. A better understanding of the variations in welfare in broiler breeders reared under commercial conditions is crucial to assess and improve the welfare situations of these birds, and to reduce the negative effects of feed restriction. However, understanding the connections between welfare and production parameters is also extremely important for animal welfare research to be able to have an impact on commercial husbandry practices. Reduced growth under stressful circumstances such as feed restriction is currently understood to be a sign of high stress and reduced welfare, however our results indicate that the actual relationship between early growth and welfare in broiler breeders may be more complex.

\section{Acknowledgments}

This study was financed in its entirety by project grant 2013-293 from the Swedish research council Formas to J Altimiras.

\section{References}

Aviagen 2013 Ross Parent Stock Management Handbook: Ross 308.Aviagen Ltd, Midlothian UK, pp 29-

Bennett CD and Leeson S 1989 Research Note: Growth of Broiler Breeder Pullets with Skip-aDay Versus Daily Feeding. Poultry Science 68: 836-838

\section{Bruggeman V, Onagbesan O, D’Hont E, Buys N, Safi M, Vanmontfort D, Berghman L, Vandesande F and Decuypere E 1999 Effects of Timing and Duration of Feed Restriction During Rearing on Reproductive Characteristics in Broiler Breeder Females. Poultry Science 78 :} $1424-1434$

Chen SE, McMurtry JP and Walzem RL 2006 Overfeeding-Induced Ovarian Dysfunction in 
Broiler Breeder Hens Is Associated with Lipotoxicity. Poultry Science 85: 70-81

Collias N and Joos M 1953 The Spectrographic Analysis of Sound Signals of the Domestic Fowl. Behaviour 5: 175-188

Dawkins MS, Cain R, Merelie K and Roberts SJ 2013 In search of the behavioural correlates of optical flow patterns in the automated assessment of broiler chicken welfare. Applied Animal Behaviour Science 145: 44-50

de Jong IC, van Voorst S, Ehlhardt DA and Blokhuis HJ 2002 Effects of feed restriction on physiological stress parameters in growing broiler breeders. British Poultry Science 43(2): 157168

Decuypere E, Bruggeman V, Onagbesan O and Safi M 2002 Endocrine Physiology of Reproduction in the Female Chicken: Old Wine in New Bottles. Avian and Poultry Biology Reviews 13(3): 145-153

Estevez I, Andersen I-L and Nævdal E 2007 Group size, density and social dynamics in farm animals. Applied Animal Behaviour Science 103: 185-204

Forkman B, Boissy A, Meunier-Salaün M-C, Canali E and Jones RB 2007 A critical review of fear tests used on cattle, pigs, sheep, poultry and horses. Physiology \& Behavior 92: 340-374 Gross WB and Siegel HS 1983 Evaluation of the Heterophil/Lymphocyte Ratio as a Measure of Stress in Chickens. Avian Diseases 27(4): 972-979

Hocking PM, Maxwell MH and Mitchell MA 1993 Welfare assessment of broiler breeder and layer females subjected to food restriction and limited access to water during rearing. British Poultry Science 34: 443-458

Hocking PM, Maxwell MH and Mitchell MA 1996 Relationships between the degree of food restriction and welfare indices in broiler breeder females. British Poultry Science 37(2): 263-278 Jackson S and Diamond J 1996 Metabolic and digestive responses to artificial selection in 
chickens. Evolution 50(4): 1638-1650

Jones RB, Satterlee DG and Marks HL 1997 Fear-related behaviour in Japanese quail divergently selected for body weight. Applied Animal Behaviour Science 52: 87-98

Katanbaf MN, Dunnington EA and Siegel PB 1989 Restricted Feeding in Early and LateFeathering Chickens. 3. Organ Size and Carcass Composition. Poultry Science 68: 359-368

Kikusui T, Winslow JT and Mori Y 2006 Social buffering: relief from stress and anxiety. Philosophical Transactions of the Royal Society B: Biological Sciences 361: 2215-2228.

Koolhaas JM, Korte SM, de Boer SF, van der Vegt BJ, van Reenen CG, Hopster H, de Jong IC, Ruis MAW and Blokhuis HJ 1999 Coping styles in animals: current status in behavior and stress-physiology. Neuroscience and Biobehavioral Reviews 23: 925-935

Korte SM, Beuving G, Ruesink W and Blokhuis HJ 1997 Plasma Catecholamine and Corticosterone Levels During Manual Restraint in Chicks from a High and Low Feather Pecking Line of Laying Hens. Physiology \& Behavior 62(3): 437-441

Leenstra FR and Pit R 1988 Fat Deposition in a Broiler Sire Strain. 3. Heritability of and Genetic Correlations Among Body Weight, Abdominal Fat, and Feed Conversion. Poultry Science 67: 1-9

Marin RH, Freytes P, Guzman D and Jones RB 2001 Effects of an acute stressor on fear and on the social reinstatement responses of domestic chicks to cagemates and strangers. Applied Animal Behaviour Science 71: 57-66

Marx G, Leppelt J and Ellendorff F 2001 Vocalisation in chicks (Gallus gallus dom.) during stepwise social isolation. Applied Animal Behaviour Science 75: 61-74

Mench JA 2002 Broiler breeders: feed restriction and welfare. World's Poultry Science Journal 58(1): 23-29

Pinchasov Y, Nir I and Nitsan Z 1985 Metabolic and Anatomical Adaptations of Heavy-Bodied 
Chicks to Intermittent Feeding I. Food Intake, Growth Rate, Organ Weight, and Body Composition. Poultry Science 64: 2098-2109

Skinner-Noble DO, Jones RB and Teeter RG 2003 Components of Feed Efficiency in Broiler Breeding Stock: Is Improved Feed Conversion Associated with Increased Docility and Lethargy in Broilers? Poultry Science 82: 532-537

Zuidhof MJ, Robinson FE, Feddes JJR, Hardin RT, Wilson JL, McKay RI and Newcombe M 1995 The Effects of Nutrient Dilution on the Well-Being and Performance of Female Broiler Breeders. Poultry Science 74: 441-456

Zuidhof MJ, Schneider BL, Carney VL, Korver DR and Robinson FE 2014 Growth, efficiency and yield of commercial broilers from 1957, 1968 and 2005. Poultry Science 93: 1-13 


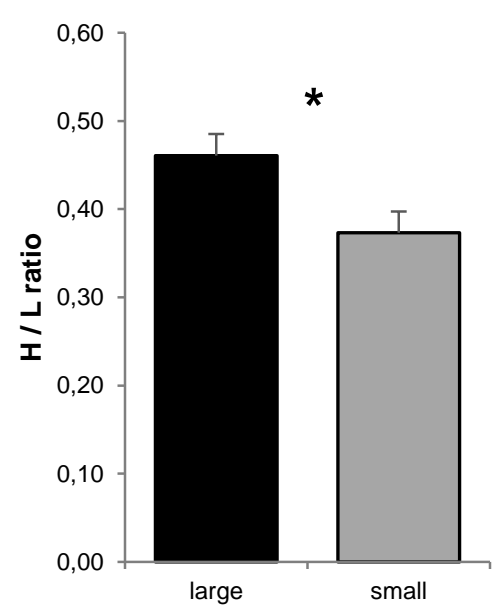

b)

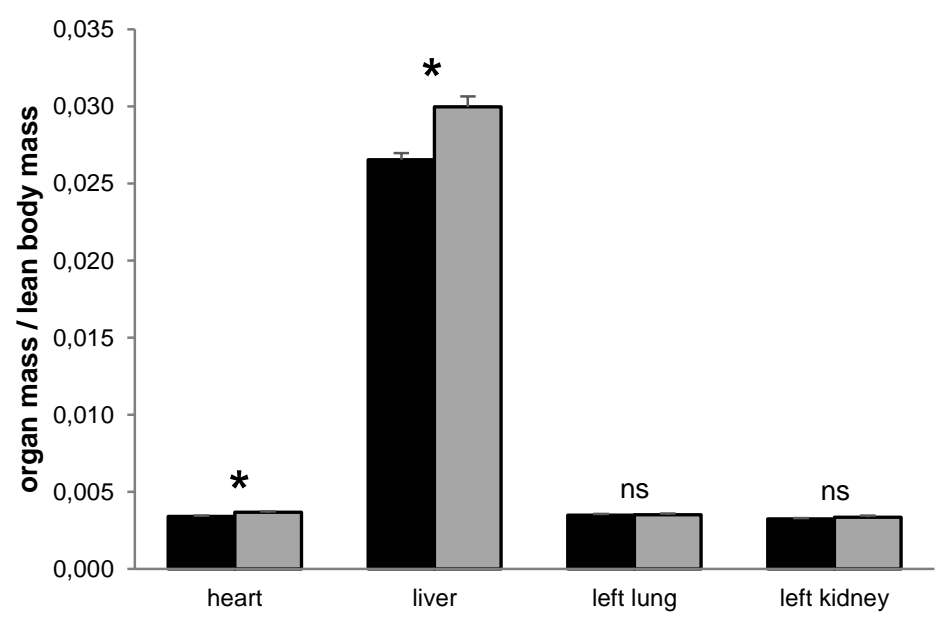

Figure 1. (a) Heterophil / lymphocyte ratios from 28 large (black bars) and 31 small (grey bars) chicks at 33 days of age. Larger birds have significantly higher $\mathrm{H} / \mathrm{L}$ ratios $(P=0.014)$, possibly indicating higher levels of perceived stress. (b) Relative organ masses of some vital organs. Hearts and livers were relatively larger in smaller animals $(P=0.002$ and $<0.001$ respectively), while relative lung and kidney weights did not differ significantly. All data analysed by Student's t-test.

a)

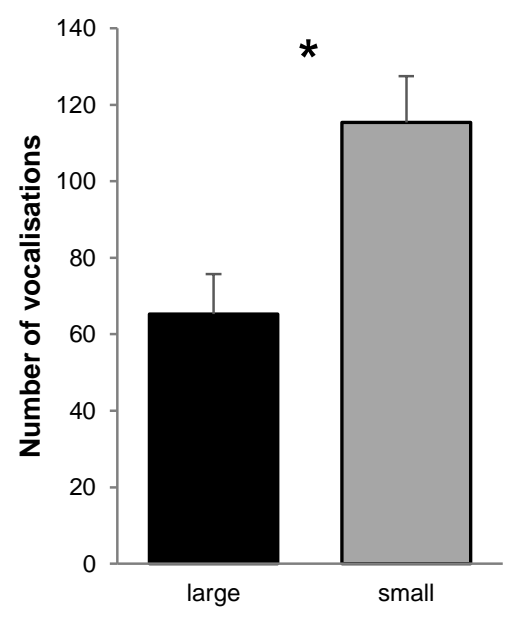

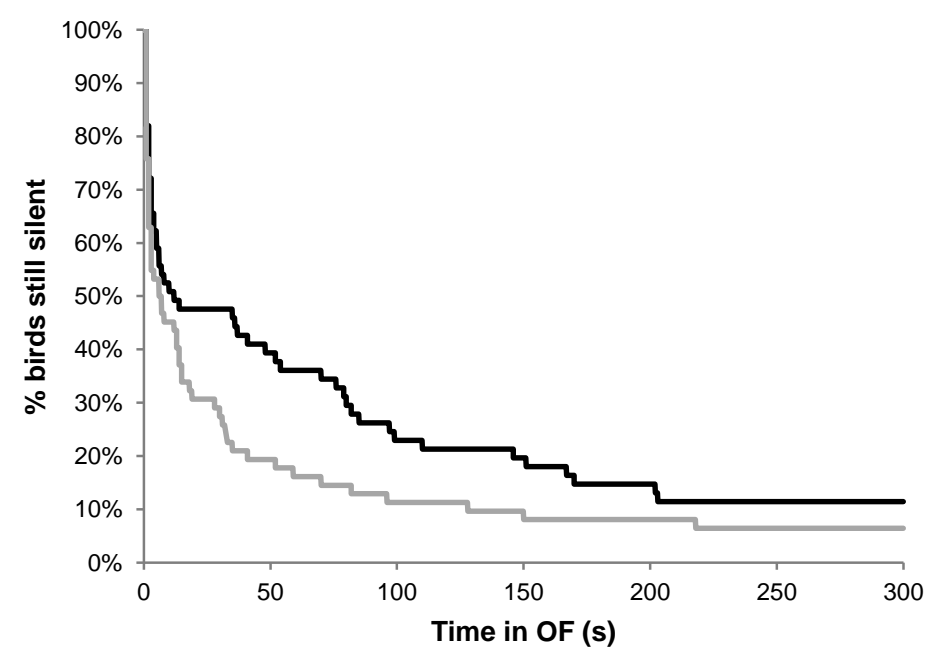

Figure 2. (a) Small (grey) birds vocalise significantly more than large (black) birds in the OF ( $P$ $=0.002$; t-test). One bird was excluded from the large group because of technical problems with the recorder. All vocalisations were categorised as either fear trills or distress calls. (b) Small birds tend to start vocalising more quickly during the OF test, but the difference is not significant $(P=0.057$; Log-Rank). 

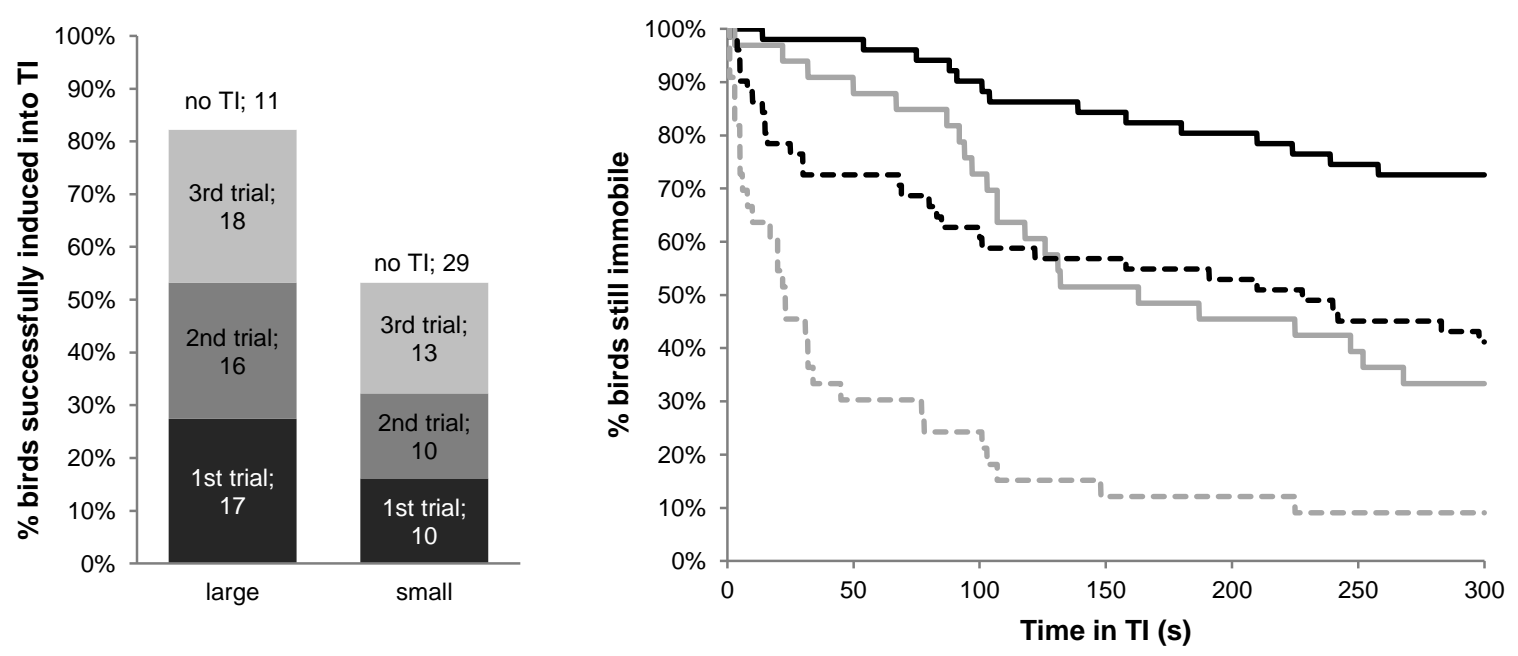

Figure 3. (a) Small birds were more resistant to TI induction $(P=0.001$; Log-Rank) and (b) showed a significantly shorter duration (small in grey) of tonic immobility (solid lines) when induced ( $P<0.001$; Log-Rank) compared to large birds (black). Latency to move the head (dashed lines) shows equivalent results $(P<0.001$; Log-Rank).

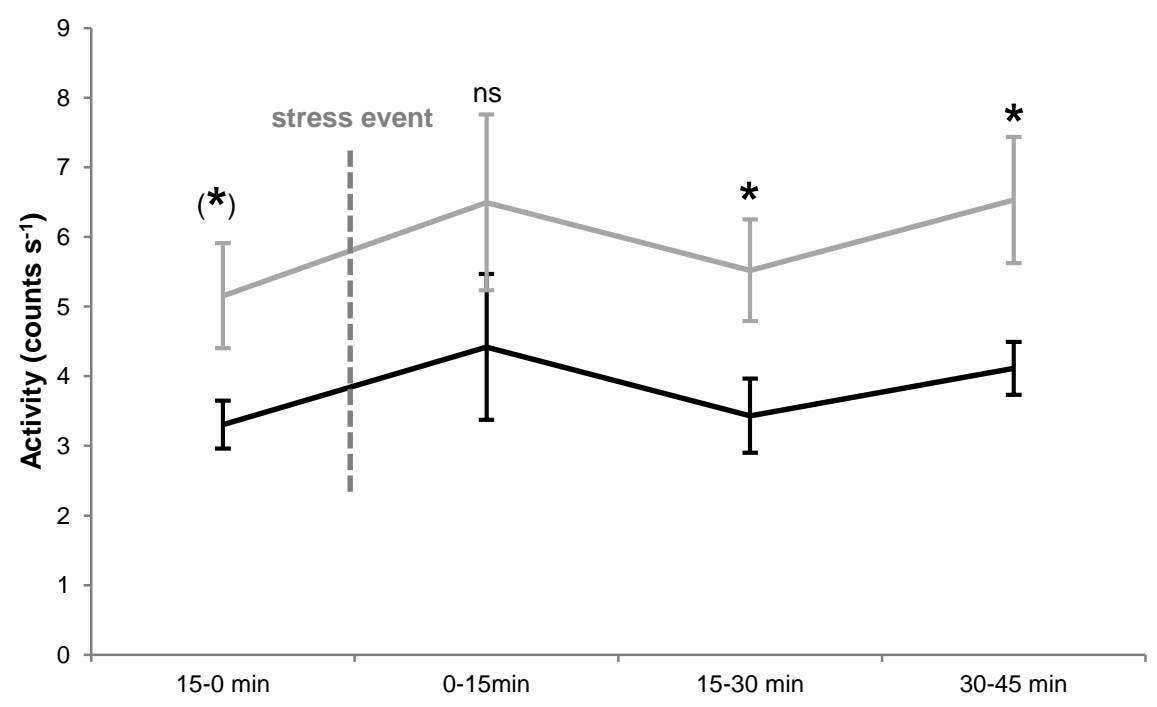

Figure 4. Home-pen activity of eight small (grey) and nine large (black) birds before and after a stressful event in the home pen, as measured by a MotionWatch 8 triaxial accelerometer strapped to their back. Time points marked as significantly different (*) have $P<0.05$ in a Student's t-test. Activity levels before experiencing the stressor ("15-0 min") borders on significance at $P=$ 0.052 . 


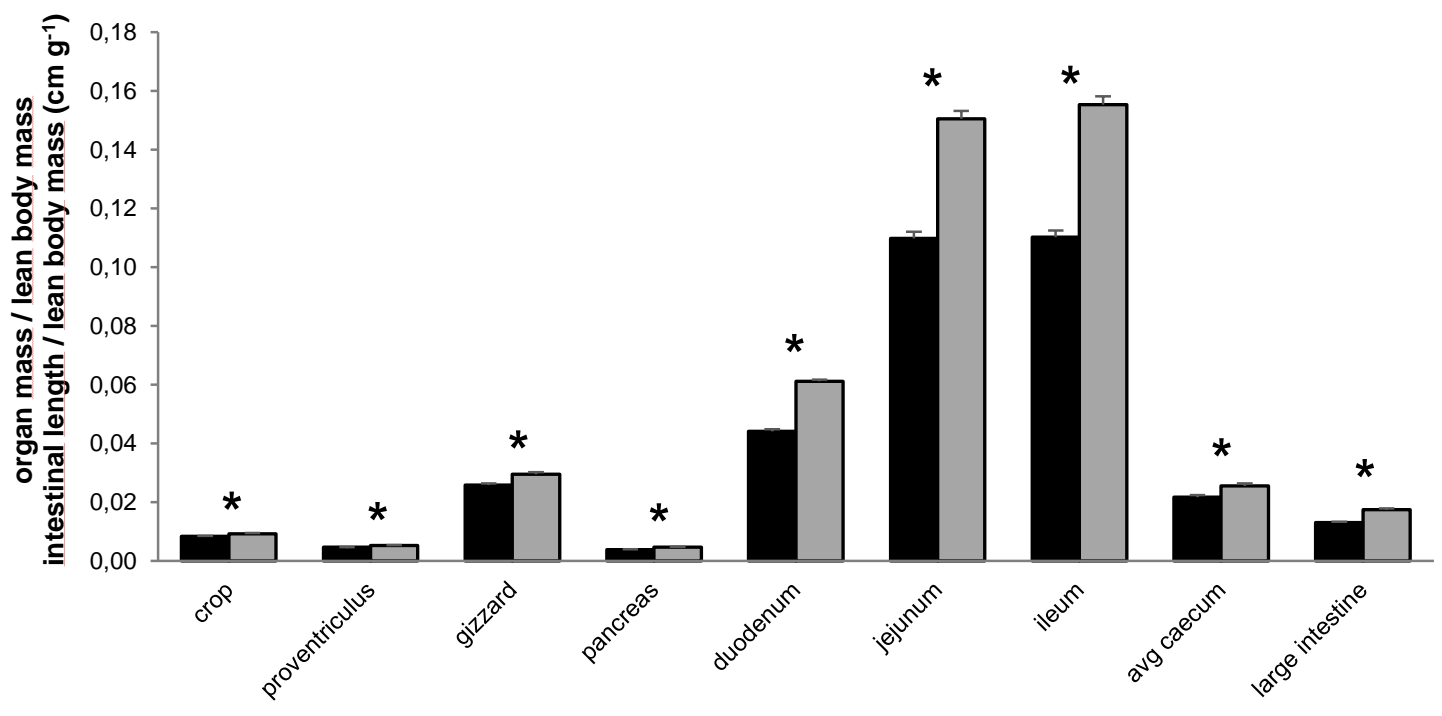

Figure 5. Organs of the gastrointestinal tract are all significantly relatively larger in smaller (grey) birds than in large (black) birds. For the large and small intestine as well as the average caecum, values are given as cm / g lean body mass. 
Table 1. All recorded organ sizes relative to lean body weight. Residual feed is the feed left in the crop, proventriculus and gizzard and is not different in absolute terms. $P$ values from Student's t-test.

\begin{tabular}{|c|c|c|c|}
\hline ORGAN & $\begin{array}{l}\text { SMALL GROUP } \\
\text { MEAN (SD) }\end{array}$ & $\begin{array}{l}\text { LARGE GROUP } \\
\text { MEAN (SD) }\end{array}$ & $P$ VALUE \\
\hline HEART & $0.37 \%(0.03)$ & $0.34 \%(0.03)$ & 0.002 \\
\hline LIVER & $3.00 \%(0.37)$ & $2.65 \%(0.23)$ & $<0.001$ \\
\hline LEFT LUNG & $0.35 \%(0.04)$ & $0.35 \%(0.04)$ & ns \\
\hline RIGHT LUNG & $0.38 \%(0.04)$ & $0.39 \%(0.05)$ & ns \\
\hline LEFT KIDNEY & $0.34 \%(0.05)$ & $0.33 \%(0.03)$ & ns \\
\hline RIGHT KIDNEY & $0.33 \%(0.04)$ & $0.32 \%(0.03)$ & ns \\
\hline SPLEEN & $0.11 \%(0.04)$ & $0.11 \%(0.03)$ & ns \\
\hline ABDOMINAL FAT & $0.19 \%(0.10)$ & $0.28 \%(0.20)$ & 0.038 \\
\hline RESIDUAL FEED & $6.02 \%(3.73)$ & $3.92 \%(2.57)$ & 0.014 \\
\hline CROP & $0.93 \%(0.13)$ & $0.84 \%(0.07)$ & 0.003 \\
\hline PROVENTRICULUS & $0.53 \%(0.06)$ & $0.47 \%(0.04)$ & $<0.001$ \\
\hline GIZZARD & $2.95 \%(0.44)$ & $2.58 \%(0.30)$ & 0.001 \\
\hline PANCREAS & $0.48 \%(0.07)$ & $0.39 \%(0.09)$ & $<0.001$ \\
\hline DUODENUM (CM /100 G) & $6.11(0.34)$ & $4.41(0.41)$ & $<0.001$ \\
\hline JEJUNUM (CM / 100 G) & $15.05(1.49)$ & $10.98(1.20)$ & $<0.001$ \\
\hline ILEUM (CM / 100 G) & $15.53(1.59)$ & $11.02(1.26)$ & $<0.001$ \\
\hline LARGE INTESTINE (CM / 100 G) & $1.75(0.20)$ & $1.31(0.21)$ & $<0.001$ \\
\hline AVG CAECUM (CM / 100 G) & $2.56(0.48)$ & $2.18(0.36)$ & 0.001 \\
\hline
\end{tabular}

